\title{
Nonlinear Optical Properties in II-VI Oxide Quantum Dot Nanostructure
}

\author{
N. Karthikeyan ${ }^{1}$, A. John Peter2,* \\ ${ }^{1}$ Department of Physics, Ramco Institute of Technology, Rajapalayam - 626 117, Tamilnadu, India. \\ ${ }^{2} P G$ and Research Deptartment of Physics, Government Arts College, Melur, Madurai - 625 106, Tamilnadu, India.
}

\section{ART ICLE DETAILS}

\section{Article history:}

Received 21 March 2018

Accepted 29 March 2018

Available online 06 April 2018

\section{Keywords:}

II-VI Material

Core/Shell Dot

Second Harmonic Generation

\begin{abstract}
A B S T R A C T
In the present work, some electronic and nonlinear optical properties are studied in a $\mathrm{CdO} / \mathrm{ZnO}$ core/shell quantum dot nanostructure taking into account the spatial confinement effect. The binding energy of an electron-hole pair is computed using Smorodinsky-Winternitz potential and the second harmonic generation is studied with the function of photon energy. The potential used in the Hamiltonian is different on both the sides of the barrier involving a two parametrical potential. The exciton binding energy is obtained employing variational method within a single band effective mass approximation and the nonlinear optical properties are probed using density matrix approach within a two level system. The larger exciton binding energies of these materials bring out the stability of electron-hole pair at room temperature. The results show that these properties are enhanced in the strong geometrical confinement region. The resonant peaks in the second harmonic generation are strongly influenced by the geometrical confinement, confined potential, matrix elements and the structural parameters. Thus, the obtained results on nonlinear optical properties of wide band gap semiconducting material will be helpful for the response to the industrial demand for optoelectronic devices operating the entire spectral region in the visible spectrum.
\end{abstract}

\section{Introduction}

Inorganic nanocrystals with certain geometries exhibit unique shape dependent phenomena and subsequent Group II-VI oxide materials are given attention because of their exotic physical and chemical properties when their dimensions are reduced and they have been attracted much due to the novel potential applications in fabricating electronic and optoelectronic devices covering the entire visible spectrum. Wide band gap oxide semiconductors are considered to be very promising candidates for semiconductor applications. It is because they have larger free exciton energy and direct band gaps. The excitonic emission continues even at room temperature and above it. These materials possess larger band gaps with higher binding energies and they can serve as light emitting diodes, laser diodes, photo-transistors, high transmittance conductive oxide coatings for solar cells, optically pumped nano-lasers, gas nanosensors and field-effect transistors [1-3]. $\mathrm{CdO}$ and $\mathrm{ZnO}$ are the promising candidates in group II-VI materials showing unique nonlinear optoelectronic elements in controlling optical signals in devices. Tailoring the band gap with size dependent optical properties, within the nanoregime, will be useful for fabricating efficient optical devices [4-6]. Nanocrystalline $\mathrm{ZnO} / \mathrm{CdO}$ composites have been prepared and the optical properties have been investigated earlier [7, 8].

In the present work, the electronic and optical properties of a wide band gap II-VI oxide core/shell quantum dot heterostructure are investigated. The spatial confinement and the incident photon energy are taken into consideration. The exciton binding energies and second harmonic generation are obtained with a new confinement potential. The variational formulism and the density matrix method have been applied to obtain those properties.

\section{Theoretical Formulism}

The taken system consists of an inner core surrounded by an outer core material. The core material is taken as $\mathrm{CdO}$ and the outer shell material is taken as $\mathrm{ZnO}$ which is the higher energy band gap than the inner material. Within the single band effective mass approximation, the Schrödinger equation, of an exciton, is obtained as

$$
\left[\hat{H}_{e x c}=\hat{H}_{e}+\hat{H}_{h}-\frac{e^{2}}{\varepsilon_{0} r}+\frac{l(l+1) \hbar^{2}}{2 m_{e(h)}^{*} r^{2}}+V(r)\right] \psi(r)=\mathrm{E} \psi(r)
$$

where $m_{e}^{*}$ is the electron effective mass and $m_{h}^{*}$ is the hole effective mass. $\varepsilon_{0}$ is the dielectric constant of the inner core material, $V(r)$ is the confined potential of the system, $l$ is the angular momentum quantum number, and $\mathrm{E}$ is the energy eigen value. The Smorodinsky-Winternitz potential is taken as the confined potential of the system. Here, height of the potential is different on both the sides of the barrier and it involves a two parameters with the following form [9]

$$
V(r)=\delta r^{2}+\frac{\lambda}{r^{2}}-\sqrt{\delta \lambda}
$$

where $\delta$ and $\lambda$ describe the materials taken in the present model. The lowest binding energies of the exciton and thereby the exciton binding energies are obtained. Using the following trial wave function, the Hamiltonian is minimized and the respective energies are found with the variation of dot size.

$$
\psi_{n l m}(r, \theta, \phi)=R_{n l}(r) Y_{l m}(\theta, \phi) \exp (-\gamma r)
$$

with $R_{n l}(r)$ and $Y_{l m}$ are the radial and the spherical harmonic wave functions respectively. Thus, the variational parameter is employed to obtain the exciton ground state energy by minimizing Eq.(1)

$$
E=\min _{\gamma} \frac{\langle\Psi|H| \Psi\rangle}{\langle\Psi \mid \Psi\rangle}
$$


The exciton binding energy of the system, using variational formulism, is given as

$$
E_{b}=E_{e}+E_{h}-\langle H(\gamma)\rangle_{\min }
$$

with $E_{e}$ and $E_{h}$ are the energy eigenvalues of the single electron and hole without the interaction term between them. $\langle H(\gamma)\rangle_{\min }$ is calculated by minimizing the (Eq.(1)) in respect of the variational parameters. Generally, it is known that the optical response of the material behaves nonlinearly when it is exposed by the intense radiation of light. The induced light wave on the semiconductor is described by the response of the electrical polarization which is a linear function of the applied electric field and it is obtained as

$$
P=\chi^{(1)} \cdot E
$$

where $\chi^{(1)}$ is the linear susceptibility. The nonlinear effect, with the high intense light, takes the form as given by

$$
P=\chi^{(1)} \cdot E+\chi^{(2)} \cdot E^{2}+\chi^{(3)} \cdot E^{3} \ldots
$$

where $\chi^{(2)}$ and $\chi^{(3)}$ are the first order and second order susceptibilities respectively. Within a three level system, the nonlinear optical properties are investigated with the incident photon energy. The second order nonlinear susceptibilities at double frequency in a three level system is given by [10]

$$
\chi_{2 \omega}^{(2)}=\frac{e^{3} N}{\varepsilon_{0}} \frac{\mu_{01} \mu_{12} \mu_{20}}{\left(\hbar \omega-E_{01}-i \hbar \Gamma_{10}\right)\left(2 \hbar \omega-E_{20}-i \hbar \Gamma_{20}\right)}
$$

where $N$ is the density of carriers in the quantum dot $E_{i j}=E_{i}-E_{j}$ and $\Gamma_{10}=\Gamma_{20}=\Gamma_{0}$ are the relaxation rates.

\section{Results and Discussion}

Numerical calculations are presented in order to investigate the electronic and optical properties of an electron-hole pair in the group II-VI core/shell quantum dot using a different kindly potential namely, Smorodinsky-Winternitzt. The spatial confinement and the incident photon energy are varied so as to obtain those properties. The atomic units are used throughout the calculations in which Planck constant is assumed, the electronic charge and the electron mass $m_{0}$ are assumed as unity. The second harmonic generation with the incident photon energy in a $\mathrm{CdO} / \mathrm{ZnO}$ core/shell quantum dot is presented in Fig. 1 . The inner radius of the core/shell quantum dot is taken as $40 \AA$. It is observed that the variation of maximum peak of plotted second harmonic generation shows two peaks one at $\omega=\omega_{10}$ and another one at $\omega=\omega_{20} / 2$, explaining the energy spacing between energy levels and of course, it is effectively affected by the structural parameters of the system involved. The magnitude of the second order susceptibility is observed to be higher energies for longer relaxation time. The resonant peaks in the second harmonic generation are strongly influenced by the geometrical confinement, confined potential, matrix elements and the structural parameters.

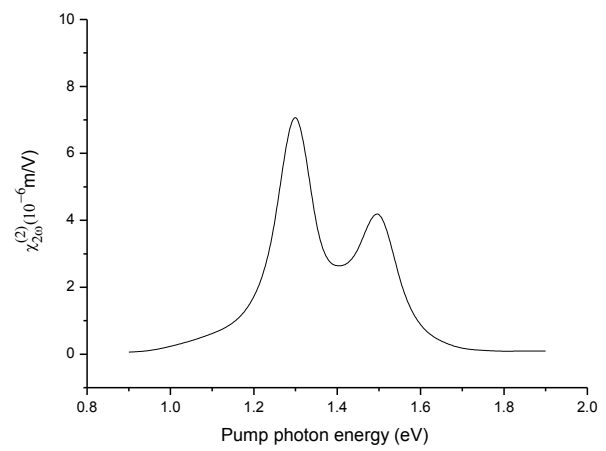

Fig. 1 Variation of second harmonic generation as a function of photon energy in $\mathrm{CdO} / \mathrm{ZnO}$ core/shell quantum dot

\section{Conclusion}

In conclusion, the electronic and optical properties of a group II-VI oxide core/shell quantum dot heterostructure have been studied. The exciton binding energy and the second harmonic generation have been obtained. The excitons play an important role in nonlinear optical properties of group II-VI oxide wide band gap semiconducting materials even at room temperature and they are useful for the potential applications in ultraviolet-blue optoelectronic nano-devices.

\section{References}

[1] K. Senthil, Y. Tak, M. Seol, K. Yong, Synthesis and characterization of ZnO nanowire-CdO composite nanostructures, Nanoscale Res. Lett. 4 (2009) 13291334.

[2] D.K. Gupta, M. Verma, K.B. Sharma, N.S. Saxena, Ind. J. Pure App. Phys. 55 (2017) 113-121.

[3] S. Wei, Q. Chang, Z. Zeng, Curr. Appl. Phy. 11(1) (2011) 16-20.

[4] L. Pavesi, L. Dal Negro, C. Mazzoleni, G. Franzò, F. Priolo, Nature 408 (2000) 440-444.

[5] E.K. Elmaghraby, M.A. Kaid, M.F. Zaki, Acta Phy. Pol. A. 125 (2014) 82-86.

[6] Q.H. Li, Q. Wan, Y.G. Wang, T.H. Wang, Abnormal temperature dependence of conductance of single Cd-doped ZnO nanowires, Appl. Phys. Lett. 86 (2005) 263101-263104.

[7] F.Z. Wang, Z.Z. Ye, D.W. Ma, L.P. Zhu, F. Zhuge, H.P. He, Appl. Phys. Lett. 87 (2005) 143101-143101.

[8] S. Meenakshi Sundar, C.K. Mahadevan, P. Ramanathan, On the preparation of ZnO-CdO nanocomposites, Mater. Manuf. Proces. 22 (2007) 400-403.

[9] P. Christina Lily Jasmine, A. John Peter, Chang Woo Lee, Intersubband optical transition energy in a $\mathrm{CdTe} / \mathrm{Zn}_{0.2} \mathrm{Cd}_{0.8} \mathrm{Te} / \mathrm{ZnTe}$ core/shell/shell spherical quantum dot with Smorodinsky-Winternitz confinement potential, Chem. Phys. 452 (2015) 40-45.

[10] J. Flórez, Á. Camacho, Excitonic effects on the second-order nonlinear optical properties of semi-spherical quantum dots, Nanoscal. Res. Lett. 6 (2011) 268274.

About the Conference: "International Conference on Nanomaterials (ICAN) - 2018" has been organized by Dr. G. Ramalingam, ICAN2018 Organizing Secretary, Assistant Professor, Department of Nanoscience and Technology, Alagappa University, Karaikudi, TN, India at his designated venue on $26^{\text {th }} \& 27^{\text {th }}$ February 2018. 